\title{
Reciprocity: A Predictor of Mental Health and Continuity in Elderly People's Relationships? A Review
}

\author{
Live Fyrand \\ Department of Research, Diakonhjemmet University College, P.O. Box 184, Vinderen, 0319 Oslo, Norway \\ Correspondence should be addressed to Live Fyrand, fyrand@diakonhjemmet.no
}

Received 25 January 2010; Revised 23 June 2010; Accepted 23 June 2010

Academic Editor: Gjumrakch Aliev

Copyright () 2010 Live Fyrand. This is an open access article distributed under the Creative Commons Attribution License, which permits unrestricted use, distribution, and reproduction in any medium, provided the original work is properly cited.

\begin{abstract}
Many studies have demonstrated that social relationships confer mental health benefits. This paper aims to identify whether and how reciprocity in social relationships predicts or is associated with mental health benefits as well as with continuity in elderly people's social relationships. The studies reviewed in this paper show that, among elders, being in a balanced or underbenefited reciprocal position predicts better mental health and life quality than being in an overbenefited position. Throughout the course of life, reciprocity evens out present and earlier reciprocal imbalances, securing continuity in close relationships-particularly between spouses and between elderly parents and adult children. In friendships, securing continuity seems to be based on the maintenance of independence based on balanced reciprocal relations, making these relationships more vulnerable. Due to the problems of conceptualization and measurement in the reviewed studies, one should be cautious in stating a final conclusion that the reciprocity norm has a universal positive effect on mental health and continuity in elderly people's relationships.
\end{abstract}

\section{Introduction}

Paradoxically, while a huge number of empirical studies have investigated the mental health benefits of being connected to social relations and social groups [1], very few studies have investigated the basics for social relationships. Reciprocity is regarded as a basis mechanism that creates stable social relationships in a person's life [2]. It has been described generally as the process of "give-and-take" - the degree of balance in social exchange between people. Nevertheless, few studies of social relations among elderly people have been undertaken in which reciprocity has been a key variable.

In addition to the changes that aging brings to people's health in general and their way of functioning in daily life, aging people usually need increased help from others due to increased age-related health problems. This may imply that they-sooner or later-will be in a disadvantaged position in the "exchange market" - most frequently being in a receiving position due to increased need for help combined with less power and prestige [3]. According to the exchange theory, being more dependent on others may cause unbalanced relationships, with associated mental distress and discontinuity as possible negative consequences. Therefore, elderly people may be more exposed to mental distress and social isolation than younger people who are less dependent on others. These age-related changes may in turn influence elderly people's social interactions, creating a need to understand possible mechanisms of these relational challenges. A better understanding of such mechanisms may help us prevent relational discontinuity and negative mental health consequences of the aging process in elderly people.

This paper examines whether and how reciprocity can predict mental health benefits or continuity in elderly people's social relations. This may represent an expansion of knowledge and tools based on a bio-psycho-social perspective for professionals working with elderly persons.

1.1. Important Theoretical Concepts. There seems to be four important concepts in the reciprocity field of theory which are interconnected in the description of important presupposition for social interaction: reciprocity, exchange, equity, and indebtedness.

The concepts of "exchange" and "reciprocity" are closely related and often used as synonyms. However, while exchange can be defined as "the action of, or act of, reciprocal giving, and receiving," reciprocity is defined as a "mutual action, 
influence, giving, and taking" [4]. Exchange of resources is by social psychologists seen as the social events most relevant to relationship formation and maintenance. Gouldner [2] regarded the norm of reciprocity as the basic assumption for social exchange interactions, viewing this norm as universal as the incest taboo and a "starting mechanism" in people's motivation to initiate contact and interaction on a utilitarian basis. The reciprocity norm determines the value and level of the content in the exchange process, implicitly setting the equivalence standard (i.e., stipulating the repayment to be "roughly equivalent" to the value of what has been received). This is meant to prevent reciprocal imbalance or inequality which further may create relational instability between the exchange partners.

While the reciprocity concept is basic describing the degree of balance of the transactions in the social exchange, is equity a more subjective assessment. The degree of reciprocity can be characterized as equitable in its fairness and justification based on the persons' values and norms. According to the equity theory is fairness in relationships with others depending on the observer's perceptions and assessments of the value and relevance of the participants' inputs and outcomes [5], being "in the eye of the beholder". Thus, it is viewed as a basic value and not as a social norm. When the amount of support received is more or less than that provided, we talk about being in an imbalanced and undesirable status [6].

There seem to be two kinds of imbalanced status: (1) an overbenefited status, meaning that a person is receiving more than giving which can be related to a feeling of indebtedness, guilt, and shame, and (2) an underbenefited status, providing more support than one receives which may lead to feelings of resentment, burden, and dissatisfaction [7]. While both equity and exchange theories suggest that individuals will be dissatisfied in underbenefited relationships (less received than provided), equity theory states that individuals also will experience overbenefited relationships as unsatisfactory, which is in contrast to exchange theory [8].

According to equity theory may lack of repayment for the help received place the receiver in a psychological state of indebtedness to the provider of help causing a threat to the individuals independence. This may lead to psychological distress for the indebted receiver [9]. Thus, equity theory assumes that receiving support/help may alter the subjective feeling whether there is an equitable balance of exchanges within a relationship. Empirical studies show that the greater the inequity between the partners, the more distress, and the harder they will try to restore equity $[9,10]$. Resisting support may also be understood as a way older people maintain their ego identity [11].

Giving, receiving, and repaying are crucial parts in three different phases in a reciprocal exchange process [12]. In unequal relationships (where outcome is unequal rewards minus costs), a feeling of indebtedness toward the donor (provider of help) may develop. While the provider of a gift most often will feel independent toward the receiver, will the receiver on the contrary often experience a dependence of the provider due to the problem of indebtedness toward the donor. Refusing gifts or the offer of help may therefore prevent the potential receivers' feelings of inequality and indebtedness towards the donor (i.e., chronic patients experiencing increased need for provision of help as the disease progresses often refuse to accept help). Indebtedness, with no immediate possibilities to be reduced, inhibits a person's help seeking [13]. Considerations of costs and of immediate opportunities for the receiver's repayment of his/her obligations seemed to be less important in close relationships where inequities will "even out" in the long run. Inequities will constantly occur in close relationships compared to more distant relationships.

Even if indebtedness derives from inequity, it differs in the way that equity theory views recipients as being motivated to restore equity to the donor-recipient relationship, whereas indebtedness theory views recipients as motivated to reduce their indebtedness to the donor. Gouldner [2] describes this by stating that "the shadow of indebtedness" will fall over the time between the provision of a benefit and the time for repayment. Greenberg [14] expresses a warning both to the "would-be donors" and to the "would-be recipients" about the attendant risks for both parties following the exchange process in a relationship. The warning should especially go to the would-be recipient, having responsibility for the problem and the help needed, thereby implying risks for an increase of indebtedness to the donor. "In our eagerness to find ways to help needy populations (such as the elderly and the handicapped), perhaps we have too often overlooked one of the most genuinely rewarding and mutually satisfying arrangements- encouraging the "needy" to give useful help as well as to receive it" [13]. Democritus (fourth century B.C.) offered the following advice: "Accept favors in the foreknowledge that you will have to give a greater return for them" [14].

The word reciprocity can also mean "inversely related", differentiating between (1) symmetrical (giving the same contributions to each other) and (2) complementary reciprocities (the contributions are not equivalent but compensatory or complementary) [15]. Even if reciprocity for the most has family and kinship as its basis [16] characterized by complementary reciprocity, is symmetrical reciprocity in particular related to friendship relationships. Balanced reciprocity (which can be basic in all relationships) is by definition more specific and short-term exchanges used especially in relation to more distant relationships (remote kin, neighbors, etc.) [17]. However, according to Sahlins [18] is balanced reciprocity basic in all relationships referring to the exchanges where there is a balance in which an equivalent of the thing received is returned within a finite-time period. Sahlins views balanced reciprocity as a third point between a continuum with positive and negative reciprocity at the two ends. This may be seen as the continuum on which all of us move in a psychological and social process toward our relationships whether it is close family, friends, or colleagues due to our age, gender and the life-challenges we are confronting during our life time. Neither our relationships nor we are static, but dynamic developmental processes from the earliest childhood until our death. 
While symmetrical and complementary reciprocity are interactions between the same people who exchange different kinds of transactions, can generalized, waived, constructed $[19,20]$, and stepwise reciprocity [21] be directed towards other people than to the providers of benefits.

While generalized reciprocity is described as an altruistic characteristic of networks where given support is not expected to be returned in the same proportion and from the same people, was constructed reciprocity mainly used where the caregiver had had a long-lasting relationship to the care recipient. This type of reciprocity form was mainly used in relation to confused and ambiguous persons where the caregiver most often had to interpret the recipient's nonverbal communication about their needs. Waived reciprocity occurred when expectations of immediate reciprocity were relinquished. The caregiver had an open-ended time period in their reciprocity assessment and some caregivers did not expect reciprocity at all $[19,20]$.

In stepwise reciprocity does the assistance from the caregiver go from the recipients to some new receiver and not back to the original provider of the support received [21]. The reciprocity will then have a kind of stepwise form where help is provided in the first step from A to B, where B in the next step covers his/her need to reciprocate for the help received from A further to person C. "Pay-it-forward" is a metaphor which may describe this kind of reciprocity.

1.2. Search Strategy and Selection Criteria. The identified studies focused mainly on the relationship between reciprocity and continuity and between reciprocity and mental health in the social relationships of older people. The empirical studies accessed for this paper were identified both by references in published articles and by a literature search using the following key words individually and in combination: "elderly", "social exchange", "reciprocity", "equity", "indebtedness", "mental health", and "continuity of social relations". The keywords for our literature search were located in the abstracts of the articles. The following OVID databases were searched: Medline, PsychINFO, and ISI Web of Science. While ISI Web of Science is an interdisciplinary database and Medline covers the general medical field, PsychINFO specifically covers the field of mental health. Both cross-sectional and longitudinal studies published in English, on elderly people aged 50 years or more, were included in this paper. All the studies identified in our literature search were included without any quality assessment procedure performed in the selection of the studies. This was because our literature search revealed a lack of research and published studies in this particular field of interest. The literature search had no limitations on publishing years. Twenty studies were identified. Twelve of these investigated the relationship between reciprocity and continuity in elders' social relations, and eight studies focused on the relationship between reciprocity and mental health. Empirical studies on relationships between reciprocity and mental health between professionals and elderly patients have not been included due to the difference in topic.

\section{Reciprocity and Relational Continuity}

2.1. Life-Course Reciprocity Evens Out Present and Prior Reciprocal Imbalances. Different kinds of social support (emotional and instrumental support, social companionship) are exchanged in different ways according to the particular relationship. For example, social support types differ between spouses, children, and friends [22]. This may imply that the reciprocity norm is practiced in different ways according to multifactor situations and therefore must be taken into account when seeking to understand the relationship of reciprocity to the continuity of elderly adults' social relationships.

Relational continuity seems to be a basic need in the lives of people and families, based on a universal and crosscultural human expectation [23]. Stable social relationships seems to be important due to the impact of social relationships on an individual's somatic and mental health $[1,24]$.

Eight studies were found focusing on the relation between reciprocity and continuity in elders' social relationships [7, 25-31].

The studies of Silverstein et al. [25] and Becker et al. [26] focused on intergenerational reciprocity between adult children and elderly parents. Silverstein et al. [25] examined why adult children provided support to their elderly parents over a 27-year period of time comprising 501 children; 416 child-mother and 317 child-father relationships living in California, USA. Becker et al. [26] investigated relationships between adult children and elderly parents in four ethnic groups in the United States. Five in-depth interviews of 270 respondents older than 50 years of age were conducted over a 5-year period. This study focused particularly on how the culture-specific conceptions of mutual assistance reflected the nature of social exchange and its role in creating continuity in family relationships.

Intergenerational reciprocity was found to be assumed for continuity of intergenerational relationships $[25,26]$. Adult children's earlier family experiences, particularly of emotional and social activities with their parents during their growing-up period, proved to be investments in reciprocal activities and support from the same children toward their elderly and retired parents when age-related needs for help, support, and company emerged among elders [25]. Therefore, children's motivation to provide social support to their older parents seems to be rooted in earlier childhood experiences with their parents. However, Becker et al. [26] found that when traditional cultural values were not reciprocated through deferential behavior by children, a breakdown in communication occurred. A breakdown of mutual assistance across generations was also found if there was a shift in the extended family away from cultural values supporting traditional family patterns.

In two longitudinal studies, Klein Ikkink and van Tilburg investigated whether reciprocity predicted continuity in elderly people's social relationships and prevented termination of elders' ties in 408 [27] and 2057 [28] elderly Dutch men and women living independently (mean age, 68 years). The respondents were drawn from the population registers of 11 municipalities representing differences in 
culture, religion, urbanization, and aging in the Netherlands. Eight types of relationships were included in their studies.

The studies of Klein Ikkink and van Tilburg demonstrated that relationships among close kin are most likely to be continued, whereas more peripheral relationships are more vulnerable to termination $[27,28]$. Over a year, unbalanced relationships became more balanced; the initially balanced ties still remained balanced, as well. However, even if being overbenefited by their close kin was experienced as an uncomfortable situation, it did not represent a threat to the continuity of the relationship, probably due to the norm that reciprocity cannot be fully applied between elderly people and their children [27]. Being overbenefited of instrumental support from family members-having a reciprocal imbalance in the present-seems to restore and even out earlier imbalances between close family members that may have been caused by past overbenefiting of instrumental support toward family. This practical help provided by close kin relationships made elderly people more independent toward their friends.

Antonucci and Akiyama [29] as well as Ingersoll-Dayton and Antonucci [7], in a cross-sectional study, investigated the perceptions of reciprocal and nonreciprocal emotional and instrumental (sick care) support in relation to spouses, children, and friends in a sample of 718 middle-aged and elderly adults $\geq 50 y$ of age in the United States, focusing on the reciprocal lifespan perspective in three different agegroups. According to the relationship type, the spousal relations were found to be most reciprocal compared with other relational types [29]. In addition the "older elders"” seemed to feel more comfortable being overbenefited by receiving instrumental support from children, taking into account a life-course perspective with previous life periods when the older persons provided family members with more support than they received. "A sense of life-course reciprocity is probably more difficult to adopt with friends or others who are known for shorter periods of time" [29].

While life-course reciprocity seems to be more difficult to practice in relation to friends or others who are known for shorter periods of time, spouses and children adopt a perspective of life-course reciprocity [7]. Even if the relationships change due to the aging of the persons involved and their independent life experiences, parents and children usually remain in each other's relational convoys [29]. With the exception of spouses, both relationships with children and friends were perceived as somewhat less reciprocal. Overall, the equity theory perspective (the need for balanced reciprocal relationships) was more important for adults in relation to their children than their spouses, where greater imbalance was tolerated.

Lewittes [30] examined the relation between reciprocity and the duration of elders' friendships in her cross-sectional study of friendship patterns and interaction skills among 169 white and black elderly women, older than 65 years of age, living in the suburban and urban neighborhoods of Long Island and the greater New York City area. This study provides interesting information about presuppositions for the development of reciprocity in friendship relationships for elderly women. Similarities in ethnic membership, social class, and age all contributed to the likelihood of a reciprocal pattern. Reciprocal relationships seemed to include both similar and complementary exchanges. Elderly women seemed to find it more acceptable to give than to receive from friends causing a reluctance to seek help or to maintain relationships when they were on the receiving end (i.e., being in an underbenefited position). A value and norm of independence prevails, implying that it is the family that the elderly woman should turn to when help is needed.

This is partly supported by the results of a longitudinal study using participant observation and interviews, conducted by James et al. [31], of 70 people over 60 years of age living in three coastal rural areas in Western Ireland. The study comprised four waves from 1978 to 1982, showing that exchanges with more remote kin and neighbors seem to be more specific and short term (i.e., balanced reciprocity) in comparison to parent/child relationships, where expectations of return by parents from children are long term and without conscious thought of present return (i.e., generalized reciprocity). Therefore, parent/child relationships may tolerate greater present imbalance than relationships that are more likely to feature constructed or generalized reciprocity, such as relationships with friends, neighbors, and more distant relations.

The importance of having a lifespan perspective to understand the relationship between reciprocity and continuity of relations in an older-adults network, were shown in these studies. The life-course reciprocity was mainly practiced in relation to spouses and children, where former underbenefitings of support were evened out in older age. Friends however, seemed not to be included in a lifecourse reciprocity process. It seems easier to give than to receive from friends according to a value of independence in friendship relationships compared to family ties. Older adults tie duration to friends seemed thereby to be more vulnerable due to present nonreciprocal exchange caused by the lack of lifecourse reciprocity.

\subsection{The Reciprocity Norm Is Practiced in Many Different} Ways. While three studies give important information about reciprocity as a prerequisite for the continuity of social relationships between caregivers and elderly patients [20, $32,33]$, one study has investigated the relation between reciprocity and time duration with different subgroups before and after entering residential care homes [34].

In a two-year followup study of 53 (22 male/31 female) spouse caregivers (mean age was 68 years for caregivers and 71 years for patients), de Vugt et al. [32] investigated relationship problems due to behavioral issues of care recipients with dementia, and mental health problems of spouse caregivers caring for patients with Alzheimer's and Parkinson's diseases. The study was part of the Maastricht Study of Behaviour in Dementia in the Netherlands. Apathy and withdrawal were the most disruptive patient behavioral problems, and hindered the caregivers' ability to share their thoughts and feelings with patients, negatively affecting the reciprocity of the relationship between the caregiver and the care recipient regardless of the sex of the caregiver. 
A cross-sectional study by Hooker et al. [33] focused on respondents living in upstate New York; respondents were 175 spouse caregivers for patients with Alzheimer's disease $(N=88: \mathrm{m} 36 / \mathrm{fm} \mathrm{52}$; caregivers' mean age: $71 \mathrm{y})$ and Parkinson's disease $(N=87: \mathrm{m} \mathrm{32/fm} \mathrm{55;} \mathrm{caregivers'} \mathrm{age:}$ $67 \mathrm{y})$. Although the results from this study mainly support the conclusions of de Vugt et al. [32], they differ with respect to the caregiver's gender. Among the Alzheimer's care group, female caregivers (wives) reported worse mental health outcomes than the male caregivers (husbands), due to loss of reciprocity as a result of cognitive deterioration in care recipients. On this basis, the researchers concluded that loss of reciprocity in marital relationships may affect women more negatively than men:

"...to the extent that women are more likely than men to fuse marital satisfaction with general well-being, they will be at a disadvantage in the dementia care giving scenario because of the loss of reciprocity. This phenomenon may be more common among women, but is certainly not specific to them." [33].

Neufeld and Harrison [20] reveal interesting findings in their longitudinal study of 22 male caregivers - 18 of them $\geq 60$ years of age-providing care to recipients (wives $\geq 60$ years of age) with cognitive impairment caused primarily by Alzheimer's disease, living in Canada. None of the men described any reciprocity in their current relationship with the care recipient. The present lack of reciprocity in the relationships between caregivers and care recipients was found to be compensated in different ways that prevented discontinuity in these relationships. Three variations of indirect reciprocity that compensated for lack of present reciprocity were identified: constructed, waived, and generalized. Constructed reciprocity (attendance to nonverbal behavior) was used by the caregivers who felt they were committed to an ongoing relationship with their wives, based on positive, long-term relationships with them prior to the onset of cognitive impairment. The lengths of their marriages varied from 27 to 60 years, and they viewed the difficulties they faced through the lens of these relationships. However, waived and generalized reciprocity were based on a view of reciprocity as a moral norm, which may be either suspended (waived) or fulfilled by contributions to a third party (generalized). The men who gave care by obligation described their feelings in this situation as burdened, stressed, angry, lonely, and frustrated.

Bear [34] investigated the relationship between social network characteristics (including reciprocity) and tie duration among the 75 of the closest family and friends of 81 new elderly RCH residents in central Florida, USA $(\geq 60$ years of age), at RCH-entry and six months later in a faceto-face interview. Reciprocity proved to have an effect on the elderly people's tie duration with their primary ties after entry into a residential care home. Although reciprocity directly affected the frequency of visits with their relatives and both visits and speaking contact (via telephone) with their closest family and friends, present reciprocity showed no general effect on tie duration with friends. Relatives and friends continued to help the residents both directly and indirectly (generalized reciprocity) by helping the closest network members to maintain their direct support of the residents. Indirect help was explained by the fact that friends were repaying the residents for past services by helping the closest ties to maintain their contact with the residents.

\section{Reciprocity and Mental Health}

\subsection{A Balanced or Underbenefited Position Predicts Better} Mental Health. In addition to the twelve previously reviewed studies on the relation between reciprocity and continuity in elders' social relations, we identified seven studies that focused on the relation between reciprocity and mental health.

The effort-reward imbalance model-which was tested in a cross-sectional study by von dem Knesebeck and Siegrist [35] on marital, parental, and unspecific relational roles-investigated a potential imbalance between effort spent ("high cost") and rewards received ("low gain") in the subjects' social exchanges. The study comprised 1290 noninstitutionalized elderly men and women $\geq 60$ years of age: 682 in Germany (mean age, 70.8) and 608 in the United States (mean age, 72.3). Study results revealed consistent associations of nonreciprocal social exchange with depressive symptoms for both genders in both samples. The risk of depressive symptoms was about twice as high among elderly men and women who reported nonreciprocity in their social exchanges compared to subjects reporting reciprocal social exchange.

Furthermore, studies on the relationship between reciprocal social exchange for health and well-being tested the associations of different types of social activities (paid work, caring, and volunteering) and well-being [36], and between social productivity (voluntary or charity work, caring for a sick or disabled adult, and provision of help to family, friends, or neighbors) and well-being [37]. According to Siegrist et al. [38], socially-productive activities are based on the social norm of reciprocity, "...in which the effort of doing the activity is made in anticipation of an equivalent reward that reflects the value of the effort involved" [36]. Data from a cross-sectional wave in 2004 of the English Longitudinal Study of Ageing (ELSA), of 5384 participants at post-state pension age ( $\geq 60$ years for women and $\geq 65$ years for men), were analyzed to examine whether participation in social activities (i.e., caring for another person) was associated with higher levels of well-being (i.e., depression), and explained by "the reciprocal nature of these activities" [36]. The study showed that reciprocal exchange had a negative association with the degree of depression regarding the activity of caring [36]. The cross-sectional study of Wahrendorf et al. [37], was also investigating the relationship between social activity (defined as social productivity, i.e., caring for a sick or disabled person and provision of help to family) and wellbeing (i.e., depression), focusing on the quality of the activity based on the notion of exchange reciprocity. The study was accomplished on 22000 participants, $\geq 50$ and from ten European countries-using data from the SHARE study 
("Survey of Health Aging and Retirement in Europe"). The study uncovered that reciprocal activity was associated with lower scores on depression both for caring and informal help. Thus, all three studies [36-38] are lending “...support to Siegrist's observations on the importance of reciprocal exchange in social relations for health and wellbeing in later life" [36].

Liang et al.'s [39] cross-sectional study of a national probability sample of 1103 elderly individuals $>65$ years of age living in different counties in the United States, Roberto and Scott's [40] cross-sectional study of 110 noninstitutionalized white elderly people $>65$ years of age living in a southwestern city in the United States, and Rook's [41] crosssectional study of 120 elderly widowed women (mean age, 72 years; range, 60 to 89 years) recruited from four senior centers in Los Angeles, California, demonstrated that underbenefited elderly persons had a decrease in psychological distress and had fewer feelings of loneliness, compared to overbenefited elderly persons who experienced the reverse situation. Nevertheless, overbenefited elderly respondents in the study by Roberto and Scott [40] reported more anger than underbenefited elderly respondents. This was explained by a substantial need for help and support and an inability to reciprocate. This type of situation may undermine elderly adults' sense of independence and self-worth due to a change in power in the elders' disfavor, compared with previous years [40]. Thus, these studies [39-41] revealed that elderly persons who were overbenefited or underbenefited were lonelier, experiencing more mental distress when compared to elderly persons whose relationships were reciprocal.

However, psychological restoration of the feeling of inequality may be due to the elderly person's "equity potential" (i.e., personal and social competence, health, income [pensions], and available support network) [42]. In a cross-sectional study of 111 Japanese-American elderly persons (mean age, 74 years; range, 60 to 90 years) in New York City, Nemoto found that the elderly people who had strong reciprocity norms and were overbenefited had less life satisfaction. This differential in life satisfaction was due to what he called a "reciprocity-burden" to reciprocate help provided earlier in life, despite the impossibility of accomplishing the reciprocity norm due to age-related life challenges. In contrast, the overbenefited elderly who were unable to return the help provided and who had a weak reciprocity norm reported higher life satisfaction and showed fewer signs of aging.

3.2. Same Pattern Identified in Studies of Other Age Groups. Due to the findings in the previously reviewed studies on elderly people, we want to give a small amount of attention in the end of this paper to seven cross-sectional studies carried out with three populations of nonelderly participants: students $[6,43,44]$, middle-aged people [4547], and parents to children with cancer [21]. These studies uncovered more or less the same pattern regarding the relationship between reciprocity and mental health, independent of the difference in the informants' life situations. Lack of reciprocity was generally associated with negative effects and poor mental health. Underbenefited as well as overbenefited students were confronted with negative feelings toward their partners, including feelings of dissatisfaction (underbenefited students) and indebtedness (overbenefited students). Feelings caused by reciprocal imbalance included feelings of indebtedness, dependency, incompetence to cope with life challenges, shame, decreased self-worth, and anger and seemed to have a negative influence on people's health.

\subsection{The Importance of Maintaining Independence. Wen-} towski's study [17] can be viewed as a "summing-up" study of the previous studies, identifying as it does a general pattern of the relationship between reciprocity and aged people. Wentowski conducted a two-year anthropological investigation to obtain an insider's perspective on the interaction of support and exchange transactions within a network of 50 elderly people (mean age, 71 years; range, 5583 years) living in the urban American South. Independence was the key concern for all the elderly people in this study. Regardless of which strategy they used predominantly, they all shared a reluctance to accept "charity". For most people, independence is "interdependence", accepting support from the network into which one has invested over a lifetime, and thereby practicing the reciprocity norm. Whatever their perception of independence, these people are using the same cultural system of meaning that surrounds the significance of reciprocity to create personal responses to the challenges of old age.

Therefore, it seems to be important to maintain independence in a variety of cultures. Against this backdrop, James et al. [31] viewed the importance of pensions for elderly people in the following way:

One of the values of pensions as a primary power resource is that they represent a stable source of income to maintain reciprocity. After land or a house is given away, reciprocal obligations will decline and dependence may increase but checks continue to be received [31].

The studies reviewed are shown in Table 1.

\section{Conclusions and Applications}

4.1. Conclusions. The aim of this paper was to identify whether and how reciprocity can predict health benefits as well as continuity in elderly people's social relations, to increase the awareness of reciprocity as a basic mechanism of health and social relations for elders in health professional's work with elderly people.

Although the cross-sectional design of some of the reviewed studies demands that we be cautious in interpreting them, they all seemed to reveal that the degree of imbalance in social relations has significant value. Reciprocal imbalance seemed to have a potential to cause mental distress as well as both dependency and indebtedness, leading to a decrease in people's ability to cope with life challenges. The studies reviewed have revealed that both overbenefited and underbenefited women and men were lonelier, experienced more mental distress, and were less satisfied with their marital relationships than those whose relationships were 


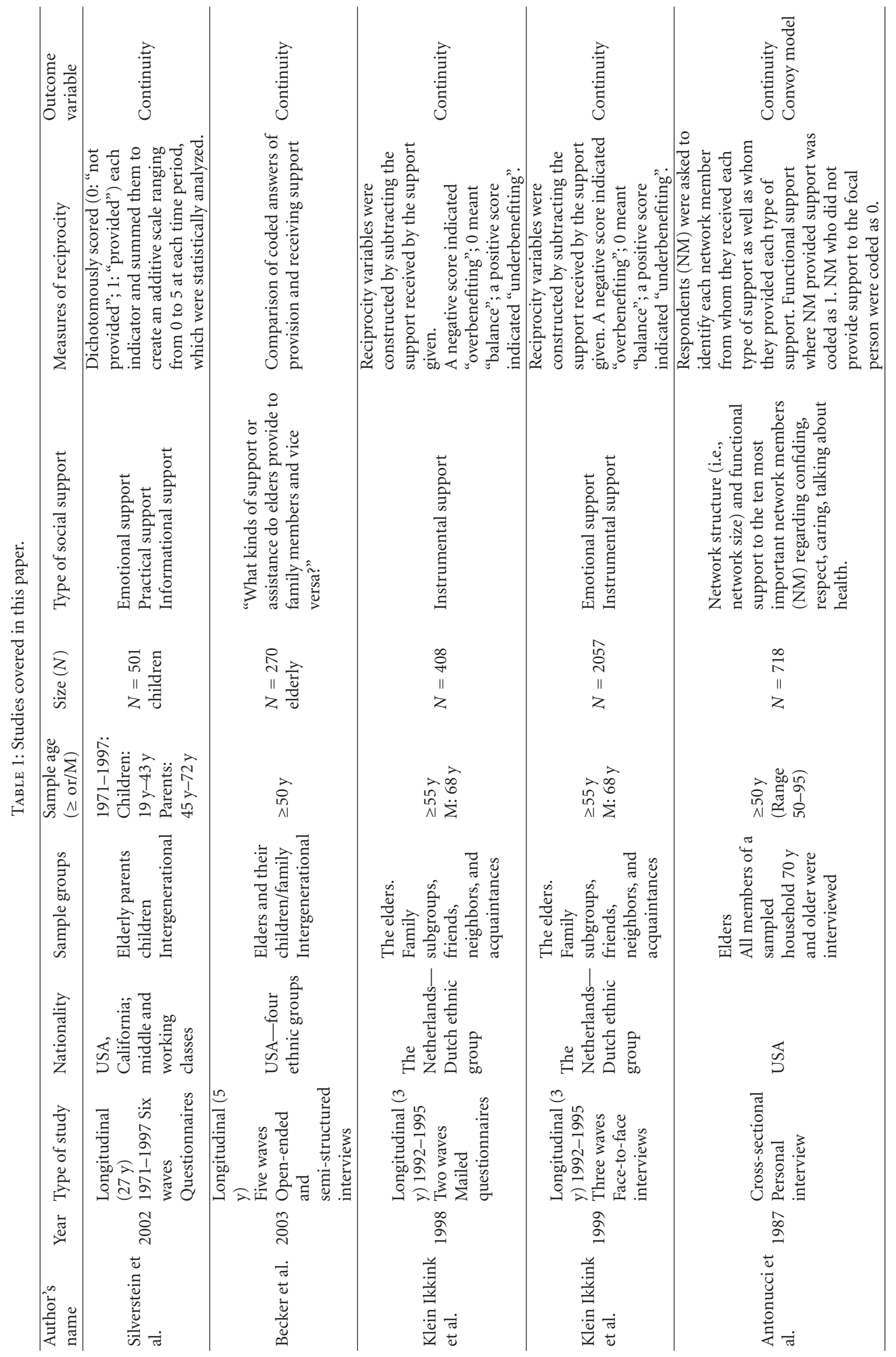




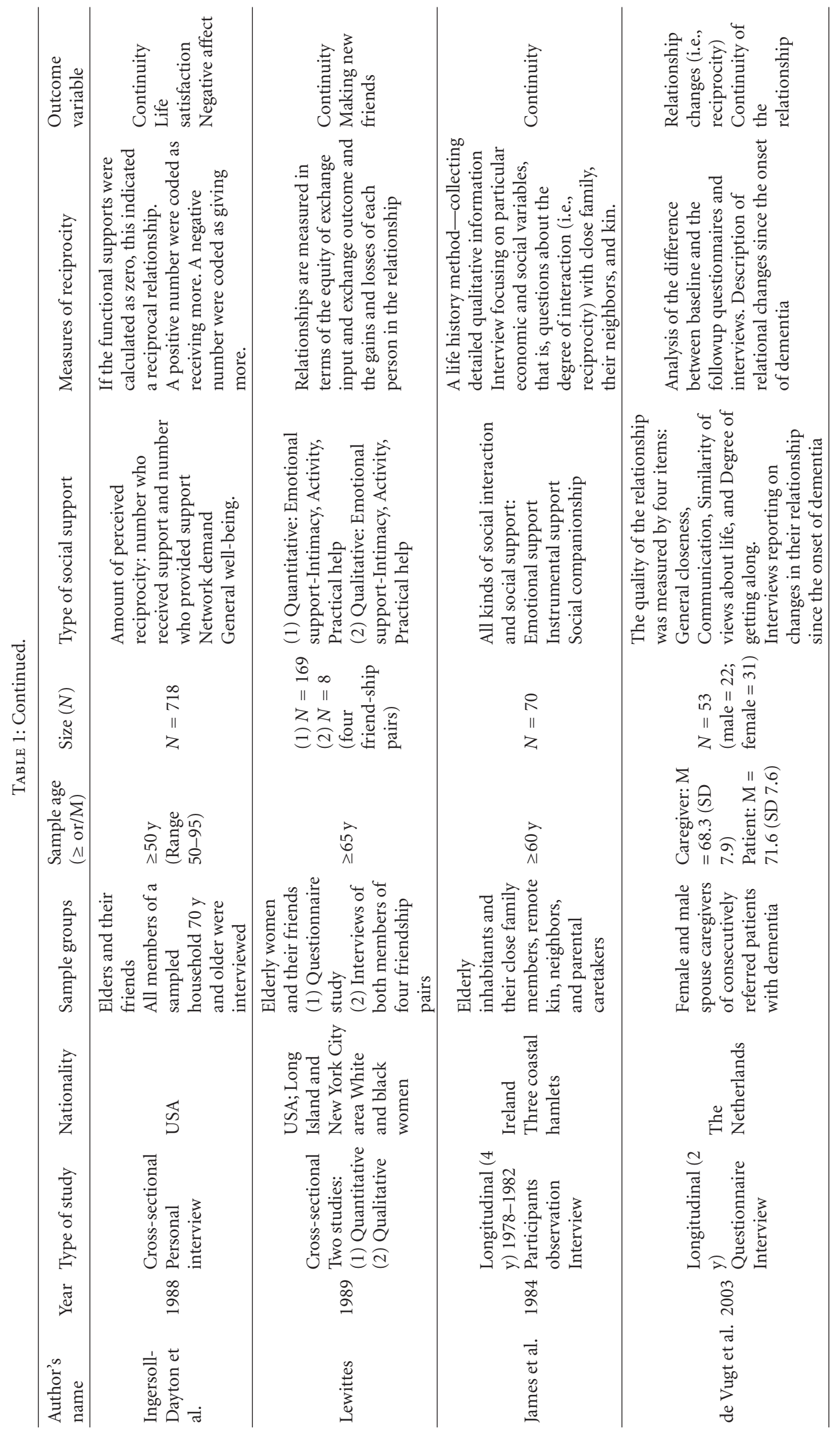




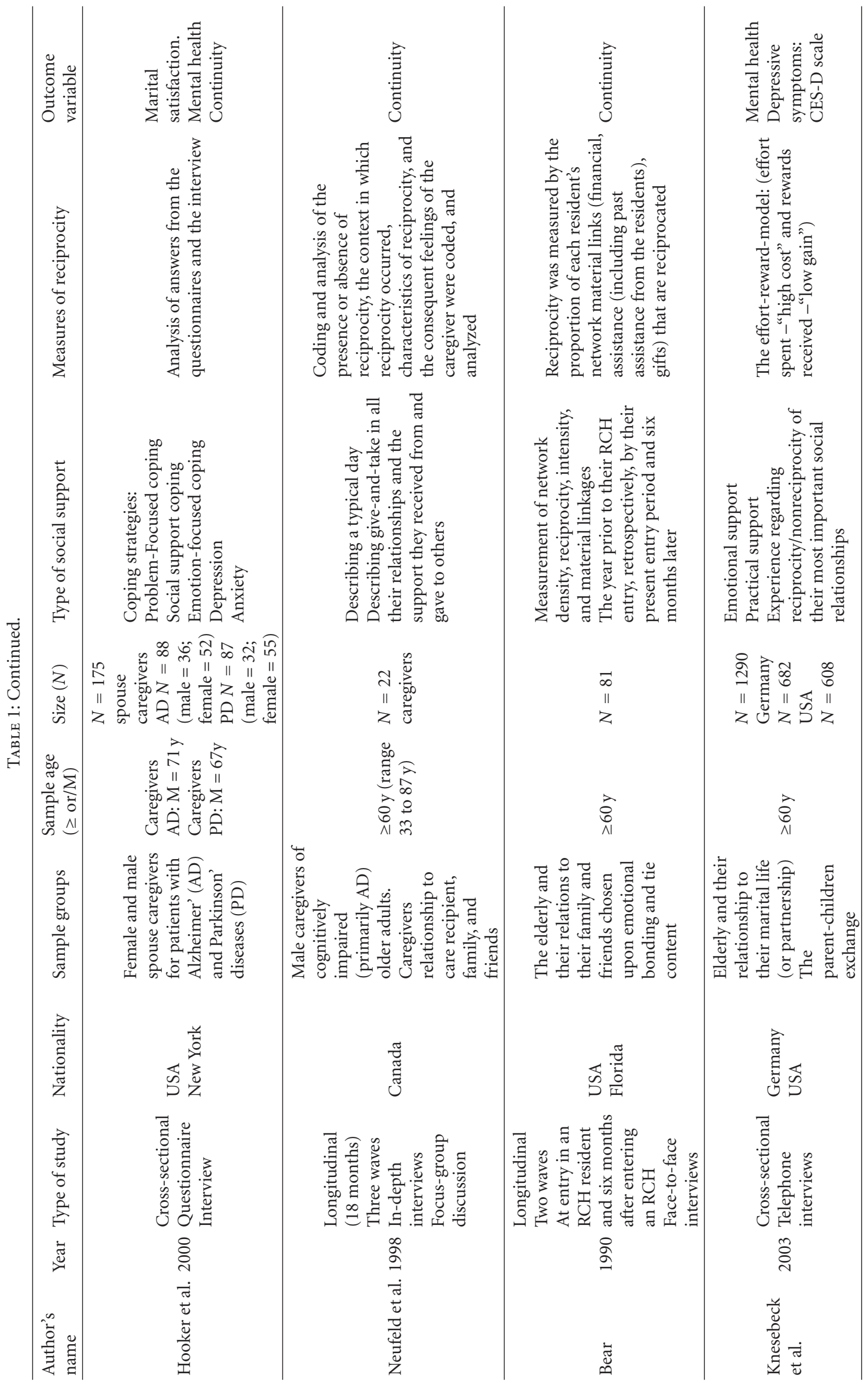




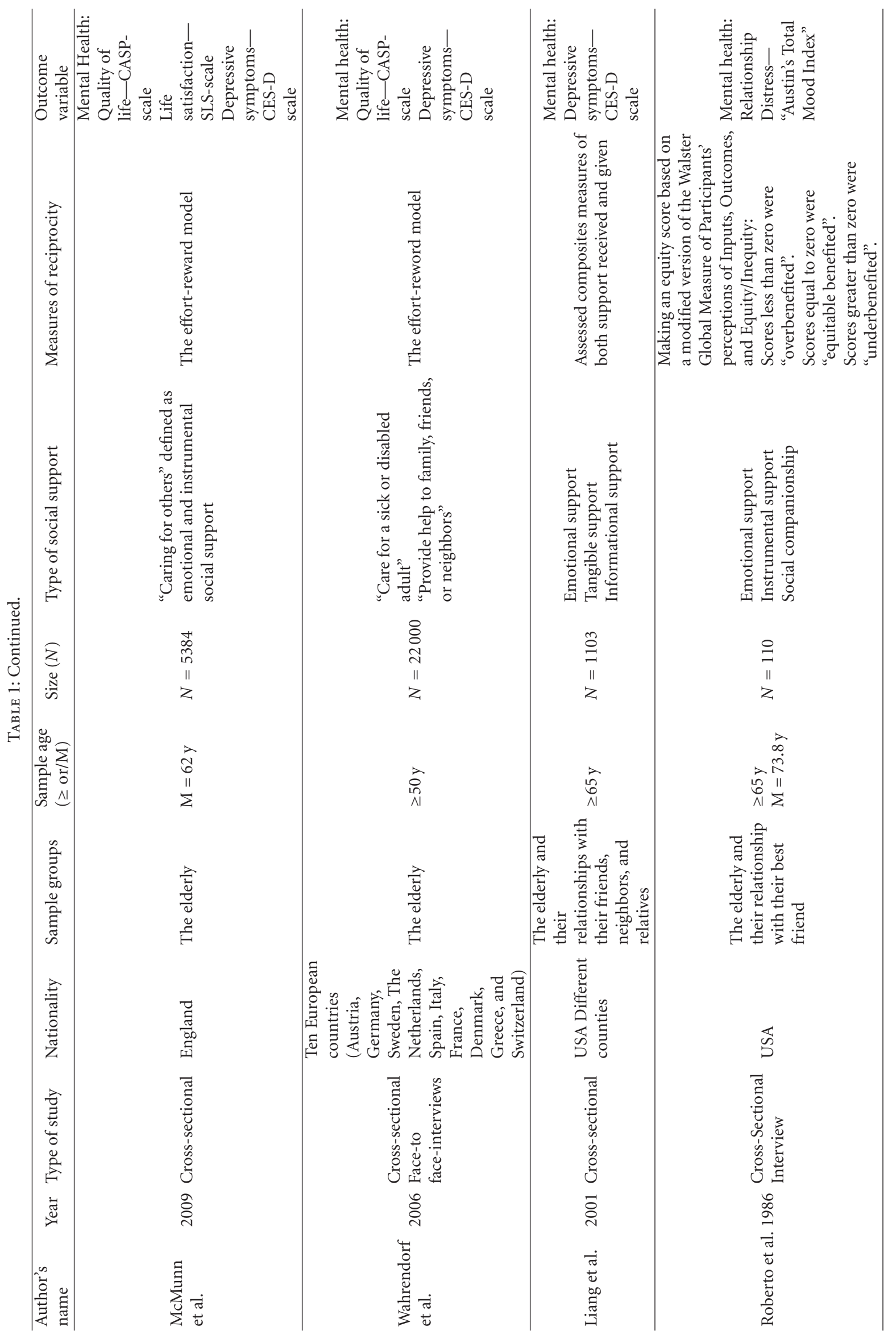




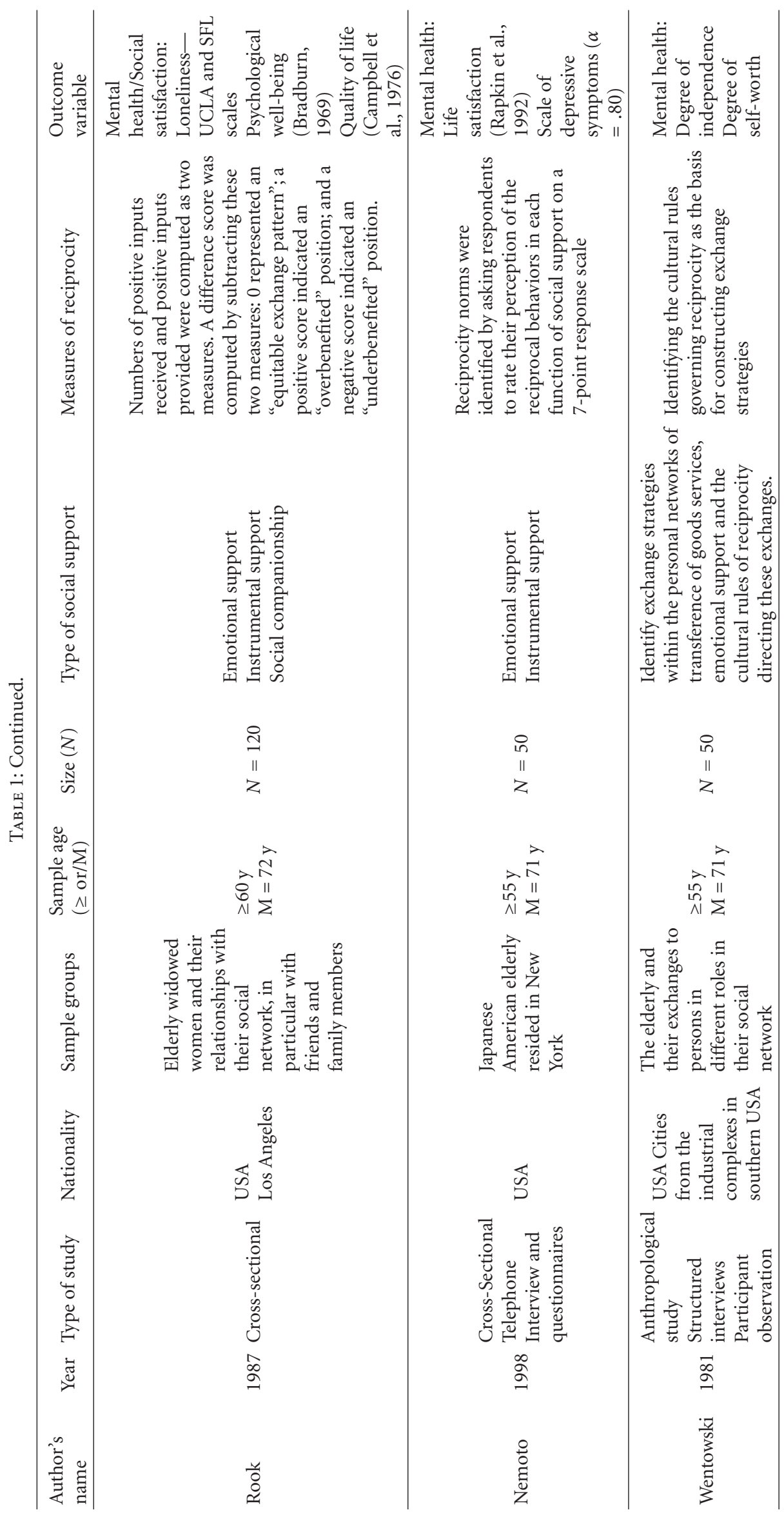


reciprocal. Nevertheless, underbenefited elderly people seem to have less mental distress and fewer feelings of loneliness than overbenefited elders.

Furthermore, this paper reveals the importance of the lifespan perspective to understanding the relationship between reciprocity and continuity of relations in an elderly adult's network. Life-course reciprocity has mainly been practiced in the context of spousal relationships and parent/child relationships, where former underbenefiting of support was balanced out in older age. Friend relationships appeared to be excluded from the life-course reciprocity process. The norm of the maintenance of independence towards friends relies on present balanced reciprocity, making these relationships more vulnerable to changes in reciprocity. In a 10-year followup study of 1477 persons aged 70 years or more, Giles et al. [48] found that "...greater networks with friends were protective against mortality in the 10 year follow up period" [48], underscoring the importance of maintaining and developing friendships.

The caregiver's role is significant from a clinical point of view. Most caregiver respondents viewed reciprocity as a moral norm that could be suspended or fulfilled by contribution to a third part, compensating for lack of reciprocity in their present relationships with care recipients. Even so, most of them felt burdened, stressed, angry, lonely, and frustrated due to not living in a close reciprocal relationship. Female caregivers seemed to be more exposed to mental health problems than male caregivers due to an association between lack of reciprocity and marital satisfaction for women. Family caregivers were unlikely to withdraw from the relationship with the care recipient even if chronic conditions disrupted care recipients' cognitive functions, causing a lack of balanced reciprocal relationships.

An interesting finding was that the cultural reciprocity norm may regulate the exchange process in social interactions, thereby decreasing possible negative consequences for elders' life satisfaction and psychological well-being. While strong reciprocity norms may increase the feelings of aging and dissatisfaction in elderly people's lives, weak reciprocity norms may decrease these feelings. Elderly persons with a weak reciprocity norm-whether individual, cultural, or both-seem to be less indebted to the caregiver. According to Jou and Fukada [6] — who studied Japanese university students_reciprocal imbalance may cause even worse health consequences in Japan than in Western societies, due to the cultural importance of the reciprocity norm that maintains the value of harmony, compared to the value of independence in Western societies.

The interpretation of the results of the studies reviewed in this paper is restricted by some limitations.

First, existing research reviewed in this paper is limited by both problems of conceptualization and measurement. The concept of social support, caring, and helping others and the type/role of relationship between the exchanging actors differs between the studies reviewed. They also vary a lot according to the research design and the measurement of the degree of reciprocity/balance in the relationships, the provision and receiving process in the exchange process $[7,29]$.
Second, some of the studies reviewed have taken a very short-term focus on issues of reciprocity and exchange, despite probable expectations that very close relationships are long-term phenomena. Adopting such a perspective may be insufficient to identify the extent of supportive exchanges across the lifespan-in particular of close relationships [8].

Third, the studies in this paper seem to reveal that reciprocity may have a significant impact on the continuity in people's relationships and on their mental health. However, the reviewed studies on reciprocity and mental health have a cross-sectional design which must be taken into account regarding the conclusions. It may be likely that health causes changes in reciprocity (i.e., a balanced, overbenefited or underbenefited position), in addition to reciprocal imbalances causing health problems. Longitudinal studies investigating causal hypotheses are therefore recommended to identify the cause-effect relationship of reciprocity to mental health.

In addition, the relatively few studies carried out regarding this paper's topics have also to be underscored.

Taken together, even if the results in the studies reviewed in this paper seem to support Gouldner's [2] statements that the reciprocity norm may be a utilitarian basis for relational stability and for a person's mental health, one should be cautious in stating a final conclusion that the reciprocity norm has a universal positive effect on mental health and continuity in elderly people's relationships.

Future research should consider using longitudinal design-in the field of reciprocity and social exchange processes for older people with the aim to identify the causeeffect relationship between the topics measured. Furthermore, the problems of conceptualization and measurements variability in this field require a more unified definition of central topics (i.e., social support, caring, and help to elderly people), classifying the elders relationships in terms of their different functions in the social network [8]. This implies that global measures (i.e., "social support") should be avoided due to the fact that they do not capture the meaning of exchange within specific relations.

Standardized measurements (quantitative as qualitative) of the multiple factors in the reciprocity process in social exchange (i.e., degree of reciprocity: a balanced or an overand underbenefited relational position) should be considered to compare results with similar studies. Measuring the multiple factors in an exchange-process seems to be important-both regarding the continuity of a functional social network and the elders mental health, due to the results uncovered in this paper. The research in the field of social network and mental health shows that a functional social network prevents social isolation and thereby a decrease of the elders mental health [1]. On this background further studies in this field should consider continuity/tie duration/longevity of social relations and mental health (life satisfaction, quality of life, mental distress, i.e., depression) as dependent variables of interest.

4.2. Applications in Clinical Work. Aging implies a transitional period where most of us gradually experience a decrease in our health and daily functioning, resulting 
in an increased need for help from others. These agerelated changes may in turn influence elderly people's social interactions, creating a need to understand possible mechanisms of these relational challenges. According to the studies reviewed here, being more dependent on others may cause unbalanced relationships that can result in mental distress and discontinuity in social relations.

Based on these findings, we will suggest that health professionals who work with elderly people incorporate the conclusions from the reviewed findings into their clinical work with the aim of maintaining elder's independence in order to prevent relational discontinuity and concomitant increases in the mental distress of elderly people. When professional helpers enter into an elder's life as professional agents they must keep in mind the challenge represented by "the helplessness-independence" paradox (i.e., increased helplessness is followed by an increased need for independence [49]. Therefore, despite elderly persons' increased need for help-the "twin needs" (the need to receive help as agerelated diseases occur and the need to maintain independence) should be noticed, both in relation to professional assistants and their personal network.

The significance of balanced reciprocal relationships for elderly persons demonstrated in this paper should be integrated as part of professionals' competence, attitude, and help provided to elderly patients, as well as into counseling and cooperating between the professional helper and the elderly's significant others. The aim should be to counsel caregivers and/or other persons in an elderly patient's life (and particularly friends) [49] to acknowledge the importance of creating or maintaining reciprocal balanced relationships and to avoid bringing an elderly patient into an overbenefited position with respect to the helper, as much as possible. Professional helpers should encourage elders and their social relations to maintain and increase reciprocal relationships, which may influence the longevity of the elder's social network, mental health, and length of survival.

\section{Acknowledgment}

The study was supported by grants from Diakonhjemmet University College for which the author is grateful.

\section{References}

[1] S. Cohen, "Social relationships and health," American Psychologist, vol. 59, no. 8, pp. 676-684, 2004.

[2] A. W. Gouldner, "The norm of reciprocity: a preliminary statement," American Sociological Review, vol. 25, no. 4, pp. 161-178, 1960.

[3] T. C. Antonucci and J. S. Jackson, "The role of reciprocity in social support," in Social Support: An Interactional View, B. R. Sarason, I. G. Sarason, and G. R. Pierce, Eds., pp. 173-198, John Wiley \& Sons, New York, NY, USA, 1990.

[4] C. A. Gregory, "Exchange and reciprocity," in Companion Encyclopedia of Anthropology: Humanity, Culture and Social Life, T. Ingold, Ed., pp. 911-939, Routledge, London, UK, 1994.

[5] E. Hatfield and S. Sprecher, "Equity theory and recipient reactions to aid," in New Directions in Helping. Vol.1: Recipient
Reactions to Aid, D. Fisher, A. Nadler, and M. DePaulo, Eds., pp. 113-141, Academic Press, New York, NY, USA, 1983.

[6] Y. H. Jou and H. Fukada, "Stress, health, and reciprocity and sufficiency of social support: the case of university students in Japan," Journal of Social Psychology, vol. 142, no. 3, pp. 353370, 2002.

[7] B. Ingersoll-Dayton and T. C. Antonucci, "Reciprocal and nonreciprocal social support: contrasting sides of intimate relationships," Journals of Gerontology, vol. 43, no. 3, pp. S65S73, 1988.

[8] A. Davey and J. E. Norris, "Social networks and exchange norms across the adult life-span," Canadian Journal on Aging, vol. 17, no. 2, pp. 212-233, 1998.

[9] M. Dunbar, G. Ford, and K. Hunt, "Why is the receipt of social support associated with increased psychological distress? An examination of three hypotheses," Psychology and Health, vol. 13, no. 3, pp. 527-544, 1998.

[10] E. Walster, G. W. Walster, and E. S. Berscheid, Equity: Theory and Research, Allyn \& Bacon, Heights, Mass, USA, 1978.

[11] C. Webb and L. Cotdingley, "Social support and renal care," EDTNA-ERCA Journal, vol. 24, no. 3, pp. 16-21, 1998.

[12] M. Mauss, The Gift, Routledge, London, UK, 1990.

[13] B. M. DePaulo, "Social-psychological processes in informal help-seeking," in Basic Processes in Helping Relationship, T. A. Wills, Ed., pp. 255-279, Academic Press, New York, NY, USA, 1982.

[14] M. S. Greenberg, "A theory of indebtedness," in Social Exchange: Advances in Theory and Research, K. J. Gergen, M. S. Greenberg, and T. A. Wills, Eds., pp. 3-26, Plenum Press, New York, NY, USA, 1980.

[15] B. Laursen and W. W. Hartup, "The origins of reciprocity and social exchange in friendships," New Directions for Child and Adolescent Development, no. 95, pp. 27-40, 2002.

[16] C. A. Gregory, "Exchange and reciprocity," in Companion Encyclopedia of Antropology: Humanity, Culture and Social Life, T. Ingold, Ed., pp. 911-939, Routledge, New York, NY, USA, 1994.

[17] G. J. Wentowski, "Reciprocity and the coping strategies of older people: cultural dimensions of network building," Gerontologist, vol. 21, no. 6, pp. 600-609, 1981.

[18] M. D. Sahlins, "On the sociology of primitive exchange," in The Relevance of Models for Social Anthropology, M. Banton, Ed., pp. 139-236, Tavistock, London, UK, 1965.

[19] A. Neufeld and M. J. Harrison, "Reciprocity and social support in caregivers ' relationships: variations and consequences," Qualitative Health Research, vol. 5, pp. 348-365, 1995.

[20] A. Neufeld and M. J. Harrison, "Men as caregivers: reciprocal relationships or obligation?" Journal of Advanced Nursing, vol. 28, no. 5, pp. 959-968, 1998.

[21] H. A. Williams, "There are no free gifts! Social support and the need for reciprocity," Human Organization, vol. 54, no. 4, pp. 401-409, 1995.

[22] L. Fyrand, T. Moum, A. Finset, L. Wichstrøm, and A. Glennås, "Social support in female patients with rheumatoid arthritis compared to healthy controls," Psychology, Health and Medicine, vol. 6, no. 4, pp. 429-439, 2001.

[23] G. G. Harris, "Concepts of individual, self, and person in description and analysis," American Anthropologist, vol. 91, no. 3, pp. 599-612, 1989.

[24] L. F. Berkman, "The role of social relations in health promotion," Psychosomatic Medicine, vol. 57, no. 3, pp. 245-254, 1995.

[25] M. Silverstein, S. J. Conroy, H. Wang, R. Giarrusso, and V. L. Bengtsor, "Reciprocity in parent-child relations over the adult 
life course," Journals of Gerontology B, vol. 57, no. 1, pp. S3S13, 2002.

[26] G. Becker, Y. Beyene, E. Newsom, and N. Mayen, "Creating continuity through mutual assistance: intergenerational reciprocity in four ethnic groups," Journals of Gerontology B, vol. 58, no. 3, pp. S151-S159, 2003.

[27] K. K. Ikkink and T. van Tilburg, "Do older adults' network members continue to provide instrumental support in unbalanced relationships?" Journal of Social and Personal Relationships, vol. 15, no. 1, pp. 59-75, 1998.

[28] K. K. Ikkink and T. van Tilburg, "Broken ties: reciprocity and other factors affecting the termination of older adults' relationships," Social Networks, vol. 21, no. 2, pp. 131-146, 1999.

[29] T. C. Antonucci and H. Akiyama, "Social networks in adult life and a preliminary examination of the convoy model," Journals of Gerontology, vol. 42, no. 5, pp. 519-527, 1987.

[30] H. J. Lewittes, "Just being friendly means a lot-women, friendship and aging," in Women in Later Years: Health, Social, and Cultural Perspectives, L. Grau, Ed., pp. 139-159, Haworth Press, New York, NY, USA, 1989.

[31] A. James, W. L. James, and H. L. Smith, ", Gerontologist, vol. 24, no. 5, pp. 483-489, 1984.

[32] M. E. de Vugt, F. Stevens, P. Aalten et al., "Behavioural disturbances in dementia patients and quality of the marital relationship," International Journal of Geriatric Psychiatry, vol. 18, no. 2, pp. 149-154, 2003.

[33] K. Hooker, M. Manoogian-O’Dell, D. J. Monahan, L. D. Frazier, and K. Shifren, "Does type of disease matter? Gender differences among Alzheimer's and parkinson's disease spouse caregivers," Gerontologist, vol. 40, no. 5, pp. 568-573, 2000.

[34] M. Bear, "Social network characteristics and the duration of primary relationships after entry into long-term care," Journals of Gerontology, vol. 45, no. 4, pp. S156-S162, 1990.

[35] O. von dem Knesebeck and J. Siegrist, "Reported nonreciprocity of social exchange and depressive symptoms: extending the model of effort-reward imbalance beyond work," Journal of Psychosomatic Research, vol. 55, no. 3, pp. 209-214, 2003.

[36] A. McMunn, J. Nazroo, M. Wahrendorf, E. Breeze, and P. Zaninotto, "Participation in socially-productive activities, reciprocity and wellbeing in later life: baseline results in England," Ageing and Society, vol. 29, no. 5, pp. 765-782, 2009.

[37] M. Wahrendorf, O. von dem Knesebeck, and J. Siegrist, "Social productivity and well-being of older people: baseline results from the SHARE study," European Journal of Ageing, vol. 3, no. 2, pp. 67-73, 2006.

[38] J. Siegrist, O. von dem Knesebeck, and G.E. Pollack, "Social productivity and well-being of older people: a sociological exploration," Social Theory and Health, vol. 2, pp. 1-17, 2004.

[39] J. Liang, N. M. Krause, and J. M. Bennett, "Social exchange and well-being: is giving better than receiving?" Psychology and Aging, vol. 16, no. 3, pp. 511-523, 2001.

[40] K. A. Roberto and J. P. Scott, "Equity considerations in the friendships of older adults," Journals of Gerontology, vol. 41, no. 2, pp. 241-247, 1986.

[41] K. S. Rook, "Reciprocity of social exchange and social satisfaction among older women," Journal of Personality and Social Psychology, vol. 52, no. 1, pp. 145-154, 1987.

[42] T. Nemoro, "Subjective norms toward social support among Japanese American elderly in New York City: why help does not always help," Journal of Community Psychology, vol. 26, no. 4, pp. 293-316, 1998.

[43] J. Jung, "The role of reciprocity in social support," Basic and Applied Social Psychology, vol. 11, no. 3, pp. 243-253, 1990.
[44] Y. H. Jou and H. Fukada, "The effects of social support reciprocity on mental and physical health of young adults," Japanese Journal of Psychology, vol. 67, no. 1, pp. 33-41, 1996.

[45] J. Griffith, "Social support providers: who are they? Where are they met? And the relationship of network characteristics to psychological distress," Basic and Applied Social Psychology, vol. 6, no. 1, pp. 41-60, 1985.

[46] T. Chandola, M. Marmot, and J. Siegrist, "Failed reciprocity in close social relationships and health: findings from the Whitehall II study," Journal of Psychosomatic Research, vol. 63, no. 4, pp. 403-411, 2007.

[47] T. Takizawa, T. Kondo, S. Sakihara, M. Ariizumi, N. Watanabe, and H. Oyama, "Stress buffering effects of social support on depressive symptoms in middle age: reciprocity and community mental health," Psychiatry and Clinical Neurosciences, vol. 60, no. 6, pp. 652-661, 2006.

[48] L. C. Giles, G. F. V. Glonek, M. A. Luszcz, and G. R. Andrews, "Effect of social networks on 10 year survival in very old Australians: the Australian longitudinal study of aging," Journal of Epidemiology and Community Health, vol. 59, no. 7, pp. 574-579, 2005.

[49] L. Fyrand, T. Moum, A. Finset, and A. Glennås, "The effect of social network intervention for women with rheumatoid arthritis," Family Process, vol. 42, no. 1, pp. 71-89, 2003. 


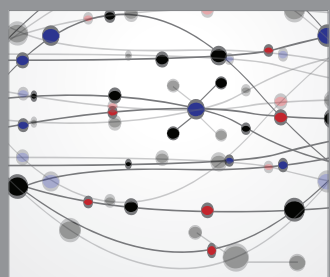

The Scientific World Journal
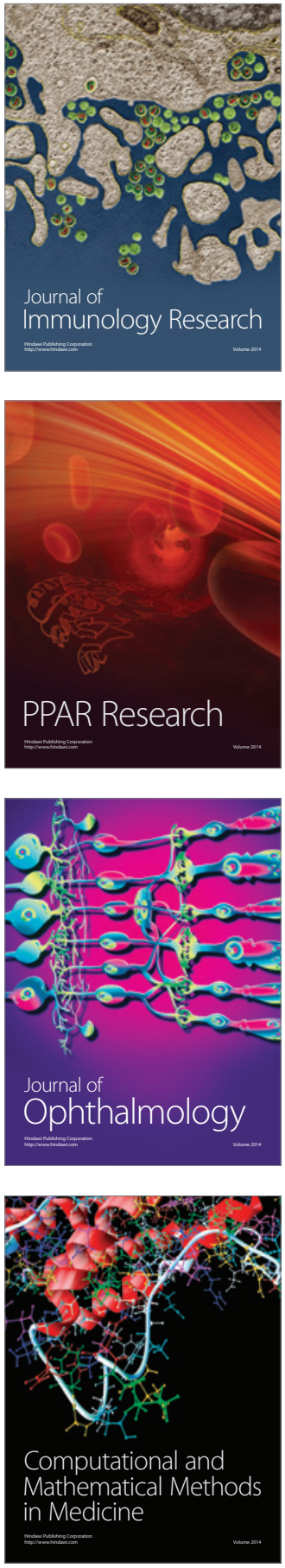

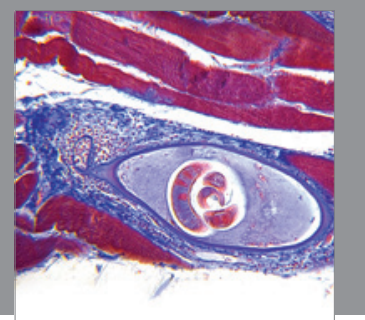

Gastroenterology

Research and Practice
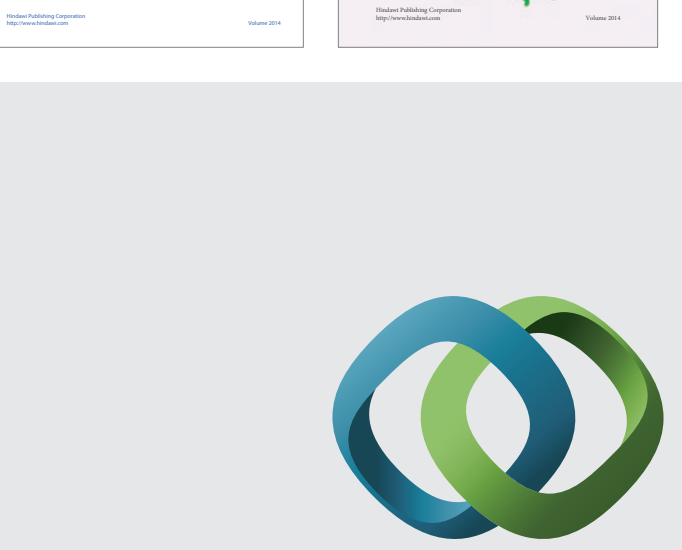

\section{Hindawi}

Submit your manuscripts at

http://www.hindawi.com
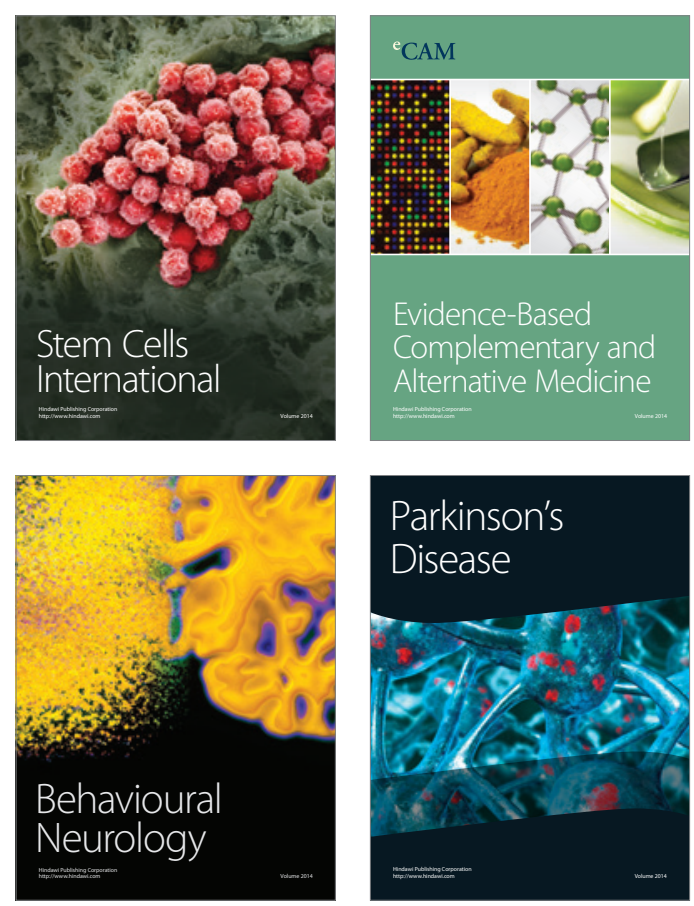

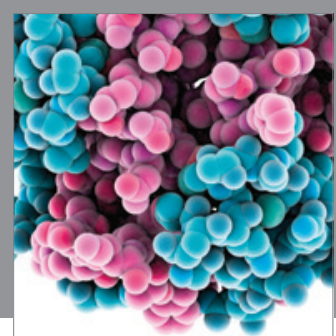

Journal of
Diabetes Research

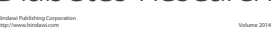

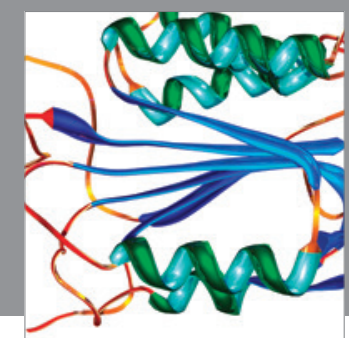

Disease Markers
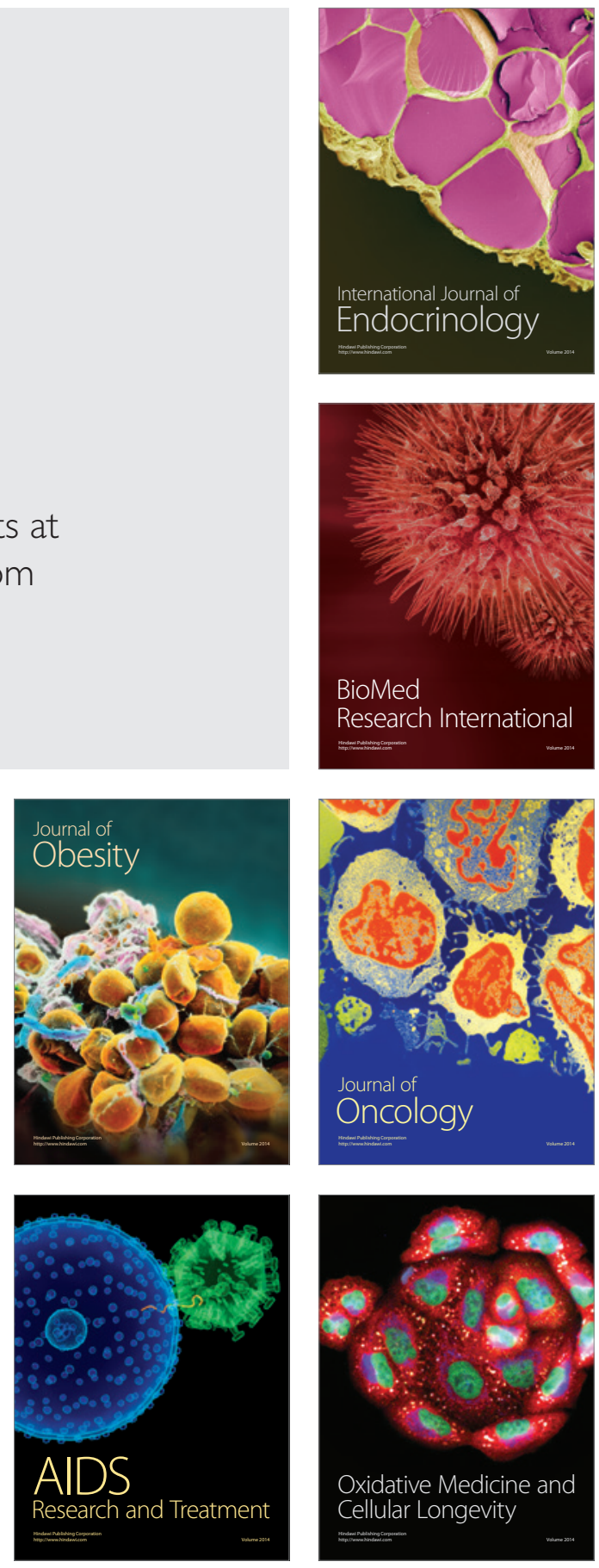